\title{
Effect of Poly Ethylene Glycol on CuO Nanoparticles and its Antibacterial Application
}

\author{
T. Hemalatha, S. Akilandeswari* \\ Department of Physics, Annamalai University, Annamalai Nagar, Chidambaram 608002. \\ Tamilnadu, India.
}

Corresponding Author* - E-mail address: akilaphy2010@gmail.com

\author{
Keywords: Effect of Poly Ethylene Glycol; CuO; Nanoparticles; Antibacterial Application
}

\begin{abstract}
Pure $\mathrm{CuO}$ nanoparticles and chemically-precipitated Poly Ethylene Glycol (PEG) used as a capping agent $\mathrm{CuO}$ nanocrystal continuum $(0.1,0.2,0.3,0.4,0.5 \mathrm{gm})$ was anatomized for structural and morphological research using X-ray diffraction (XRD), Fourier transform infrared spectroscopy (FT-IR) and Field - Emission Scanning Electron Microscopy (FE-SEM). Their X-ray Diffraction (XRD) analysis manifested monoclinic crystallinity in pure and PEG-capped CuO nanorods, with an average crystallite size of $21.63 \mathrm{~nm}$ and $13-16 \mathrm{~nm}$ respectively. The morphological analysis revealed their structural conformation. The FT-IR spectrum affirmed the presence of $\mathrm{Cu}-\mathrm{O}$ bonds. The optical property of the aforesaid nanorods was studied by UV-Visible reflectance (UVVis DRS). The UV analysis showed that all the capped products show signs of good optical quality in the UV region and also the absorption edge was blue shifted with a band gap of $1.85 \mathrm{eV}$ for $0.4 \mathrm{gm}$ PEG capped as results of quantum confinement effect. The antibacterial properties of the asprepared nanostructures investigated for various human pathogens using disc diffusion method. The result showed the significant antibacterial activity both gram positive and gram negative bacteria.
\end{abstract}

\section{INTRODUCTION}

There has been a universal increase in common interests toward nanotechnology with progression in efficient nanomaterials which are extensively used in consumer and medical products. Because of the materials at nanoscale level behave in a different ideal way and posses ideal qualities from their other equivalents; it is the main reason for the significant succession in nanotechnology $[1,2]$. Micro and nano structures have a well defined shape and inner structure because of their novel and fresh properties and diverging applications, when the size reduced to the nanometer regime, its properties changes considerably. However, the materials are customized at atomic level to reach its ideal properties such as high surface-to-volume ratio and quantum size effects and thus it can be tailored for the required applications [3]. The aggregations of nanoparticles are changes their size, shape and alter the chemical, physical and biological properties. It is therefore necessary to prevent the aggregation of the nanoparticles and to stabilize them at the desired size which can be achieved using chemical capping agents. Thus capping techniques, also called as stabilizers, plays an essential role in the synthesis of nanoparticles with reverence to size and shape control. The capping agents contain some functional groups which can ligate the metal nanoparticles [4]. However, there has been limited work on copper oxide nanorods and nanowires $[5,6]$. Hence the present work was proposed at synthesizing the $\mathrm{CuO}$ nanoparticles with PEG as a capping agent and without capping agents. Among the a choice of metal oxides, $\mathrm{CuO}$ (p-type semiconductor with band gap of $1.2 \mathrm{eV}$ ) is of immense importance owing to its wide applications in heterogeneous catalysis, gas sensing, lithium electrode material, solar cells, reactive oxidizers in nanothermite composites, and so forth. $\mathrm{CuO}$ has also been predictable as attractive oxygen carrier for capturing and parting of $\mathrm{CO}_{2}$ with little energy loss in chemical looping combustion of fossil fuels and waste to diminish the greenhouse gas emissions. [7]

Thi My Durg Dang et al., stated that PEG works as size controller and also as a surfactant of nanoparticles [8]. PEG is commonly used as a surfactant to prepare nanomaterials and as a preservative of metal colloids because of its availability, low expenditure and non toxicity. Apart 
from usage of PEG as a surfactant, PEG is used as one of the finest non-ionic polymers in biomedical science [9]. $\mathrm{CuO}$ nanorods were obtained through the reaction of cupric dodecyl sulfate $\left(\mathrm{Cu}(\mathrm{DS})_{2}\right)$ and $\mathrm{NaOH}$ at $80{ }^{\circ} \mathrm{C}$ using a solution phase synthesis was case out by Qi Liu et al [10]. Ultrasound assisted shape regulation of $\mathrm{CuO}$ nanorods in ionic liquids and their use as energy efficient lubricant additives were investigated by Rashi Gusain et al., [11].

The potential work done with some resistant bacteria strains, the antibacterial activity involving both pristine and PEG capped $\mathrm{CuO}$ nanorods for their unique properties has upheld to be of great worth and has yielded more major attractions as a possible one for the concern caused by hospital contagion harmful microorganisms are essentially caused by both Gram positive and Gram negative microorganisms such as B.subtilis, S.aureus, P.vulgaris, E.coli and V.cholerae. There-fore there is an increasing demand for novel antibacterial agents, and recently, due to their high antibacterial activity metal nanoparticles have attracted the attention of medical microbiologists world-wide. Although, unlike silver and copper antibacterial activity of copper oxide is not being studied extensively.

Among many processes, for large range production, simple chemical precipitation method has been considered to be the most on the go method as it is behind to low cost, high capacity and fine potential for high quantity production [12].To analyze the manipulation of PEG as a capping agent in pure $\mathrm{CuO}$ nanoparticles, a thumping attempt has been made by varying the concentration of the capping agent on the structural and morphological parameters on synthesized $\mathrm{CuO}$ nanoparticles and to study its antibacterial activity.

\section{EXPERIMENTAL METHODS}

\subsection{Chemicals}

Copper acetate monohydrate $\left(\mathrm{Cu}\left(\mathrm{CH}_{3} \mathrm{COO}\right)_{2} \cdot \mathrm{H}_{2} \mathrm{O}\right)$, sodium hydroxide $(\mathrm{NaOH})$ pellets and polyethylene glycol $\left[\mathrm{HO}\left(\mathrm{C}_{2} \mathrm{H}_{4} \mathrm{O}\right) n \mathrm{H}\right](\mathrm{PEG}, M \mathrm{~W}$ : 8000) were purchased from Merck, India and used as received exclusive of any further purification. Since they were of analytical reagent grade with $99 \%$ purity. De-ionized water was used all the way through the synthesis and ethanol was used for the washing purpose.

\section{Bacterial used:}

The antibacterial activity of prepared nanoparticles were investigated against five strains of Gram positive Bacterial strain namely Staphylococcus aureus (NCIM 2901), Bacillus subtilis (NCIM 2063) and gram negative bacterial strains such as Proteus vulgaris (NCIM 2027), Escherichia coli (NCIM 2256) and Vibrio cholarae (ATTC 14033) was obtained from National Collecting Industrial Microorganism (NCIM), Biochemical Sciences Division, National Chemical laboratory, Pune.

The stock cultures were maintained on nutrient agar medium at $7{ }^{\circ} \mathrm{C}$ for $24 \mathrm{~h}$, antibacterial activity was determined by using Muller Hinton Agar (MHA) and Muller Hinton Broth (MHB) obtained from Himedia Ltd, Mumbai.

\subsection{Synthesis of $\mathrm{CuO}$ nanostructures}

For the synthesis of pristine $\mathrm{CuO}$ nanocrystals, $1.99 \mathrm{~g}(0.2 \mathrm{M})$ of copper acetate in $50 \mathrm{ml}$ of deionised water was allowed to stirrer and then $1.2 \mathrm{~g}(0.6 \mathrm{M})$ of sodium hydroxide $(\mathrm{NaOH})$ pellets were mixed drop by drop to the above prepared solution. The intact mixture was stirred magnetically at $60^{\circ} \mathrm{C}$ until a homogeneous solution was obtained and also it was stirred constantly until a black precipitate was produced. The obtained dispersions were purified by washing next to de-ionized water and ethanol alternately a number of times to eliminate impurities and followed by revelation in oven to get a dried nanoparticles. In order to obtain the pure crystalline rods the dried particles were annealed for 2 hours at $400^{\circ} \mathrm{C}$.

\subsection{Synthesis of PEG capped $\mathrm{CUO}$ nanocrystals}

In the synthesis process, $0.2 \mathrm{M}$ of copper acetate in $50 \mathrm{ml}$ deionised water was stirring in magnetic stirrer. Then diverse weights $(0.1,0.2,0.3,0.4$ and $0.5 \mathrm{gm})$ of PEG were added to the above solution. The mixture was stirred magnetically at $60^{\circ} \mathrm{C}$ until a homogeneous solution was 
obtained. After that $0.6 \mathrm{M}$ of $\mathrm{NaOH}$ pellets was added drop by drop to the above mixture and stirred magnetically until a black precipitate was formed. The precipitate was washed, dried and then annealed as discussed in the above progression. Fig. 1 shows the flowchart of the preparation process of $\mathrm{CuO}$ nanoparticles.

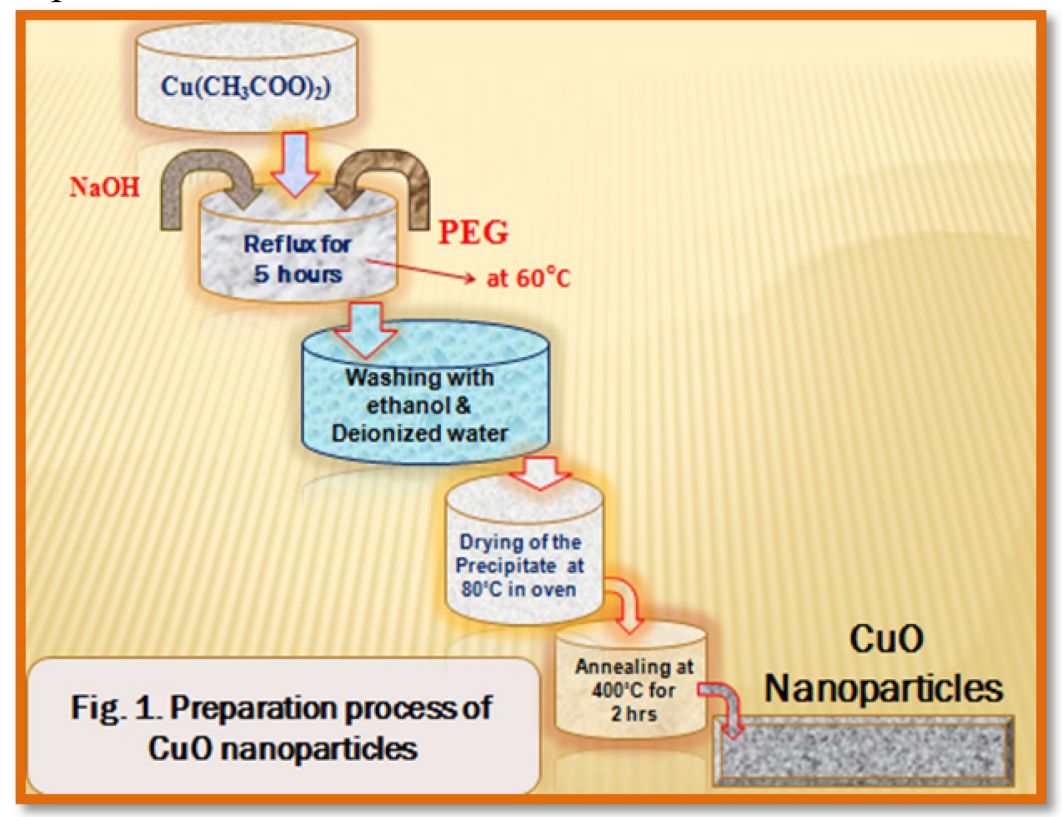

Fig. 1 Preparation process of $\mathrm{CuO}$ nanoparticles

\subsection{Characterization}

The crystal structure and phase purity of the as synthesized products were investigated by Xray diffractometer (X' PERT PRO) with $\mathrm{Cu} \mathrm{K} \alpha$ radiation $(\lambda=1.5406 \AA)$. FTIR have been employed to find the presence of functional groups in the range of $4000-400 \mathrm{~cm}^{-1}$ and it was recorded using SHIMADZU-8400 with a resolution of $4 \mathrm{~cm}^{-1}$. Measurements were performed with pressed pellets which were made using $\mathrm{KBr}$ powder as diluents. UV visible spectrum was monitored with JASCO UV V-670 Spectrophotometer to know the optical property of the synthesized samples. The morphology of the products was examined using Philips Field Emission Scanning Electron microscopy (FESEM). The antibacterial activity was done using disc diffusion method.

\subsection{Antibacterial assays}

\section{Disc diffusion method}

The antibacterial activity of compounds was determined by disc diffusion method according to Bauer et al. (1966) [13] with modification. Petri plates were prepared by pouring $20 \mathrm{~mL}$ of MHA for bacteria and then the plates were allowed to solidify and used in susceptibility test. The standard inoculum using bacterial suspensions containing $10^{8} \mathrm{CFU}$ per $\mathrm{mL}$ was swabbed on the top of the solidified respective media and allowed to dry for 10 minutes. The prepared nanoparticles were dissolved in 10 per cent Dimethyl sulfoxide (DMSO) and under aseptic conditions, sterile discs were impregnated with compounds of $200 \mu \mathrm{g} / \mathrm{disc}$. The discs with compounds were placed on the surface of the medium with sterile forceps and gently pressed to ensure contact with inoculated agar surface. Ciproflaxin $(5 \mu \mathrm{g} / \mathrm{disc})$ for bacteria was used as positive control and 10 per cent DMSO was used as blind control. Finally, the inoculated plates were incubated at $37^{\circ} \mathrm{C}$ for $24 \mathrm{~h}$ for all the bacterial strains. The zones of inhibitions were observed and measured in millimeters. The assay in this experiment was repeated three times.

\subsection{Micro dilution broth assay}

\section{Determination of the minimum inhibitory concentration (MIC) for bacteria}

The MIC of prepared nanoparticles were determined in MHB by using a microtitre plate assay as reported by Hammond and Lambert (1978) [14] $100 \mu \mathrm{L}$ of Sterile MHB for bacteria were transferred in to each well of sterile 96 micro titer well plate. The both samples were dissolved in 10 
percent DMSO to obtain $400 \mu \mathrm{g} / \mathrm{mL}$ (compounds) stock solutions respectively. A volume of $100 \mu \mathrm{L}$ of prepared nanoparticles stock solution was added into the first well. After fine mixing of the compounds and broth $50 \mu \mathrm{L}$ of solution was transferred to the second well and in this way, the serial dilution procedure was continued to a twofold dilution to obtain concentrations like 200 to $7.81 \mu \mathrm{g} / \mathrm{mL}$ (compounds) of each well. Finally, $5 \mu \mathrm{L}$ of bacterial suspension was added to each well to achieve concentrations of approximately $5 \times 10^{5} \mathrm{CFU} / \mathrm{mL}$. Each plate was a setup positive control (bacterial suspension adding $10 \mu \mathrm{L}$ of MHB) and negative control (10\% DMSO and bacterial culture). The plates were incubated at $37^{\circ} \mathrm{C}$ for $24 \mathrm{~h}$ for all the bacterial strains. The lowest concentration occurred was taken as the MIC value.

\section{RESULT AND DISCUSSION}

\subsection{Structural analysis}

The XRD patterns of uncapped and altered weights of PEG capped $(0.1,0.2,0.3,0.4$ and $0.5 \mathrm{gm}) \mathrm{CuO}$ nanocrystals are shown in Fig. 2. The obtained diffraction planes value is in good coordination with the JCPDS card No: 89-5899. It is also confirmed the monoclinic structure of $\mathrm{CuO}$ nanoparticles.

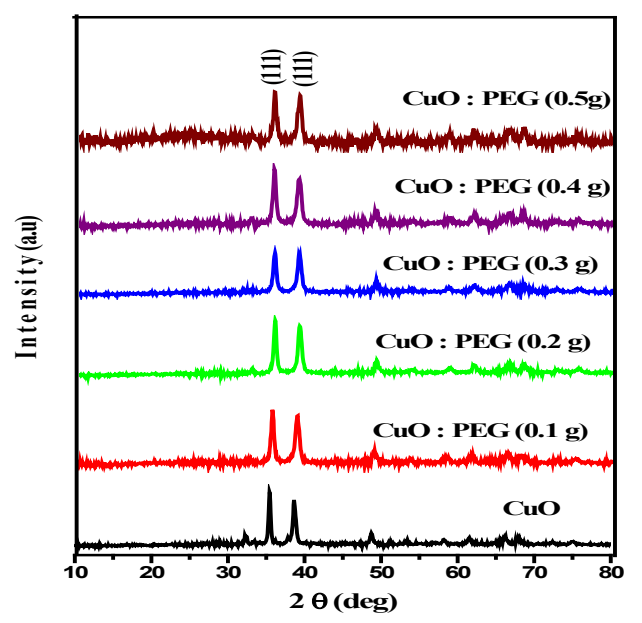

Fig. 2 XRD spectrum of Pure and Various concentration of PEG capped CuO nanocrystals

XRD pattern show no peaks of impurity with the cleared spectrum. From the XRD pattern the crystalline size were estimated for as-prepared samples. From the X-ray diffraction peak widths, the diameter of the nanocrystals was estimated through Scherrer formula [15]. The average size of the particles estimated is $21.63 \mathrm{~nm}$ for uncapped. However, the estimated sizes of the capped particles are $16.32,15.85,13.97,13.06$ and $14.21 \mathrm{~nm}$ for capping concentrations of $0.1,0.2,0.3,0.4$ and $0.5 \mathrm{gm}$ respectively. When compared with uncapped artefact, all the capped particles show reduced size as a result of capping. The mechanism behind the formation of size reduced particle is explained as follows. After introducing polymer into their action mixture, $\mathrm{Cu}^{2+}$ ions form a complex with PEG, ensuing in particle capping in the lead nucleation [16].

\subsection{Functional Analysis}

To understand the role of PEG on the synthesis of $\mathrm{CuO}$ nanoparticles, FT-IR spectra were recorded in the range between 4000 and $400 \mathrm{~cm}^{-1}$. Fig. 3 illustrates the FT-IR spectra of uncapped and diverse weights of PEG capped $\mathrm{CuO}$ nanocrystals. All the samples exhibit a broad absorption band in the range of $3330-3500 \mathrm{~cm}^{-1}$ due to stretching vibration of $\mathrm{OH}$. 


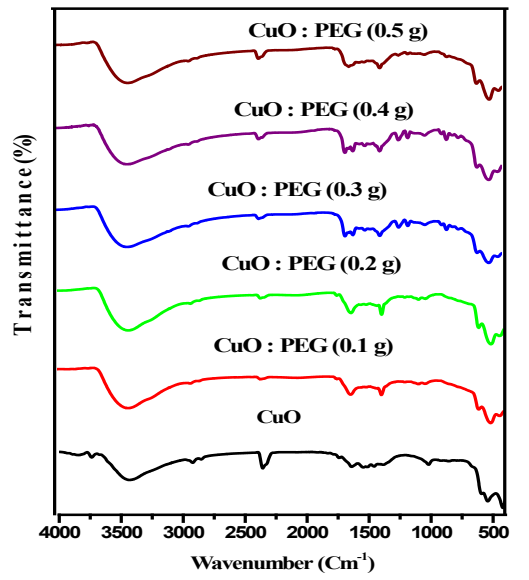

Fig. 3 FTIR spectrum of Pure and various concentrations of PEG capped nanorods

The band at $2360 \mathrm{~cm}^{-1}$ assigned to carboxylic group ( $\mathrm{COO}^{-}$) vibration in which the intensity was little high for uncapped and it gets decreases on adding the weights of PEG from 0.1gm onwards, and reached optimum at $0.4 \mathrm{gm}$ of capping of $\mathrm{CuO}$ nanoparticles. The presence of a peak at $1456 \mathrm{~cm}^{-1}$ and around $1650 \mathrm{~cm}^{-1}$ corresponding to $\mathrm{C}=\mathrm{C}$ stretching and $\mathrm{C}=\mathrm{N}$ stretching respectively. As shown in fig. 3, PEG capped samples show absorption bands at $2920 \mathrm{~cm}^{-1}$ due to $\mathrm{CH}$ asymmetric stretching vibrations. The absorption band positioned at around $2860 \mathrm{~cm}^{-1}$ is resulted from characteristic peak of PEG. The band centred at $1247 \mathrm{~cm}^{-1}$ originates from C O C bands of PEG [17]. The absorption bands indicate around 420, 530, and $590 \mathrm{~cm}^{-1}$ are due to the $\mathrm{Cu}-$ $\mathrm{O}$ stretching in the monoclinic structure of $\mathrm{CuO}[18]$.

\subsection{Optical property}

\subsubsection{UV-Vis spectroscopy}

The UV-Visible spectroscopy of the pristine and PEG capped $\mathrm{CuO}$ nanocrystals were shown in the figure 4. From the figure, it is well-known that the peak position has no change with reverence to the capping concentrations.

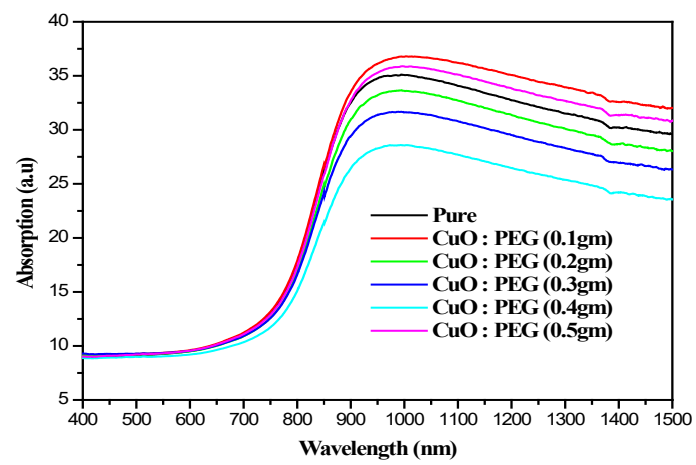

Fig. 4 UV-Vis DRS spectra of Pristine and PEG capped nanoparticles

However, on 0.4gm of PEG capping, the absorption edge was blue shifted with a band gap of $1.85 \mathrm{eV}$ as a result of quantum confinement effect. Quantum confinement effect occurs in the case of the nanoparticles when the particle size becomes as good as with or slighter than the exciton Bohr radius. All the capped products show signs of good optical quality in the UV region. As the concentration of PEG inclusion increases, the absorption edges are blue shifted with an intensity reduction in the reflectance due to quantum confinement effect. The band gap of pure $\mathrm{CuO}$ is found to be $1.76 \mathrm{eV}$; whereas the values of PEG capped $\mathrm{CuO}$ with $0.1,0.2,0.3,0.4$, and $0.5 \mathrm{gm}$ of PEG ions are about $1.80,1.82,1.84,1.85$, and $1.72 \mathrm{eV}$, respectively. Up to $0.4 \mathrm{gm}$ addition of PEG the band gap gets increases, after that the effect of capping will get increase and thus the band gap gets decreases. From this it can be observed that $0.4 \mathrm{gm}$ of PEG capped nanorods may be considered as the optimum capping level of $\mathrm{CuO}$ nanocrystals. 


\subsection{Morphological Analysis}

\subsubsection{FESEM}

Figure 5(a, b) shows the FESEM images of pure and 0.4 gm PEG capped nanoparticles. The FESEM image of uncapped $\mathrm{CuO}$ sample illustrates the formation of $\mathrm{CuO}$ nanorods with less degree of aggregation and its length of typically about $100-250 \mathrm{~nm}$. The vary in morphology of the $\mathrm{CuO}$ nanoparticles as a role of PEG capping is evidenced by FESEM image presented in fig 6(b). The flower-like nanostructures consisted of nanorods has been observed and also the rods are joined together to form Plate let like formation with its length typically about 50 to $250 \mathrm{~nm}$ was observed in PEG capped $\mathrm{CuO}$ nanoparticles.

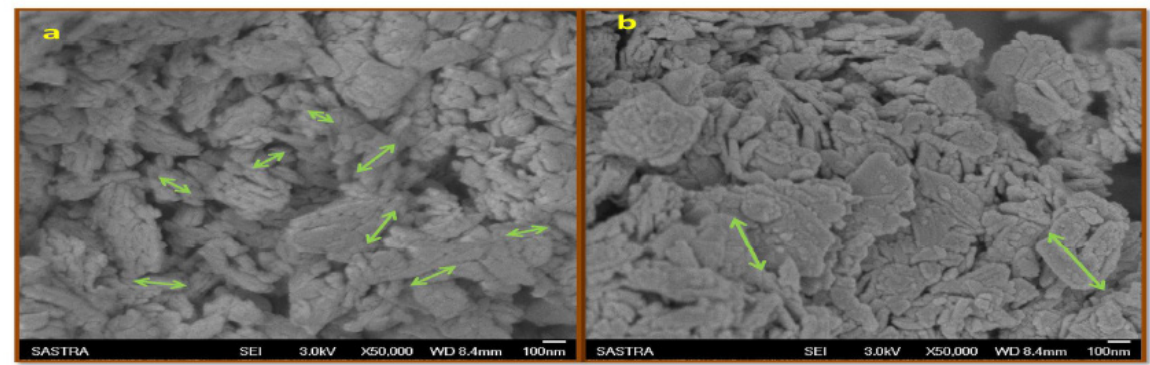

Fig. 5 FE-SEM images for uncapped and 0.4gm PEG capped nanorods

Here PEG plays a considerable role in preventing the flocculation of particles, controlling the particle size and its morphology. FESEM data established that PEG addition has a substantial effect on the morphology and size distribution of the obtained material.

\subsection{Antibacterial Activity}

Antibacterial testing for antibacterial activity revealed that both the prepared nanoparticles acted as an excellent anti bacterial agents for both gram positive and gram negative bacteria which is shown in the fig. 6 .

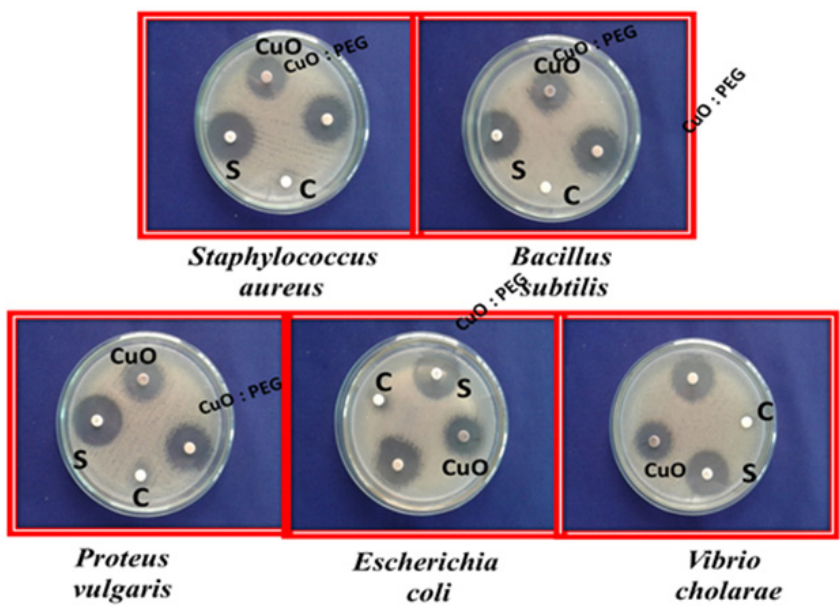

Fig. 6 Antibacterial activity photograph of different bacteria for pristine $\mathrm{CuO}$ and 0.4gm PEG capped $\mathrm{CuO}$ nanoparticles.

The activity was studied against gram +ve bacteria strains namely Bacillus subtilis and Staphylococcus aureus and gram -ve bacteria strains namely Proteus vulgaris, Escherichia coli and Vibrio cholera. The bacterial inhibition zones for $\mathrm{CuO}$ nanorods for both pure and PEG capped $\mathrm{CuO}$ nanoparticles are shown in the Table. 2 and it was compared with standard Ciproflaxin. From the table it was proved that the prepared nanoparticles exhibit a remarkable activity against human pathogens which is near to the value of standard. The highest mean zone of inhibition of $31 \mathrm{~mm}$ was developed against B. subtilis and E.coli for PEG capped $(0.4 \mathrm{gm}) \mathrm{CuO}$ nanorods. For all the bacterial strains comparing pristine, PEG capped nanoparticles shows the better zone of inhibition. Several studies have suggested two possible mechanisms for the interaction between the bacteria and the nanoparticles. The primary reason is the production of increased levels of reactive oxygen 
species (ROS). The oxygen species are mostly in the form of hydroxyl radicals and singlet oxygen. The next reason is the deposition of the nanoparticles on the surface of bacteria [19]. In addition, the MIC values of nanoparticles against all the five pathogens are listed in Table. 1 . The results of the lowest MIC values of the $\mathrm{CuO}$ nanostructures ranged between 6.25 and $25 \mu \mathrm{g} / \mathrm{ml}$.

Table 1. Antibacterial activity of synthesized $\mathrm{CuO}$ nanoparticles for Pristine $0.4 \mathrm{gm}$ capped CuO nanoparticles

\begin{tabular}{|c|c|c|c|c|c|c|c|}
\hline \multirow[b]{2}{*}{ S.No } & \multirow{2}{*}{$\begin{array}{c}\text { Name of the } \\
\text { bacterial strains }\end{array}$} & \multicolumn{4}{|c|}{$\begin{array}{c}\text { Mean zone of inhibition } \mathbf{a}(\mathrm{mm}) \mathrm{b} \\
\text { Concentration of the disc }(200 \mu \mathrm{g} / \mathrm{disc})\end{array}$} & \multicolumn{2}{|c|}{$\begin{array}{c}\text { MIC } \\
(\mu \mathrm{g} / \mathrm{disc})\end{array}$} \\
\hline & & $\mathrm{CuO}$ & $\begin{array}{c}\text { CuO: PEG } \\
(0.4 \text { gm) }\end{array}$ & 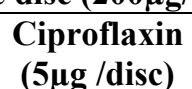 & Control & $\mathrm{CuO}$ & $\begin{array}{c}\text { CuO:PEG } \\
(0.4 \text { gm) }\end{array}$ \\
\hline 1. & Bacillus subtilis & $25.3 \pm 0.57$ & $31.3 \pm 0.57^{*}$ & $35.1 \pm 0.28$ & - & 25 & 6.25 \\
\hline 2. & $\begin{array}{l}\text { Staphylococcus } \\
\text { aureus }\end{array}$ & $26.6 \pm 0.38$ & $30.0 \pm 0.50$ & $28.3 \pm 0.57$ & $7.0 \pm 0.50$ & 12.5 & 6.25 \\
\hline 3. & Proteus vulgaris & $25.5 \pm 0.50$ & $28.6 \pm 0.76$ & $29.0 \pm 0.57$ & $8.5 \pm 0.50$ & 25 & 12.5 \\
\hline 4. & Escherichia coli & $27.3 \pm 0.47$ & $31.8 \pm 0.26$ & $29.5 \pm 0.50$ & - & 25 & 12.5 \\
\hline 5. & Vibrio cholarae & $25.1 \pm 0.28$ & $29.8 \pm 0.78$ & $31.1 \pm 0.18$ & $8.2 \pm 0.37$ & 25 & 12.5 \\
\hline
\end{tabular}

${ }^{\mathrm{a}}$ - diameter of zone of inhibition $(\mathrm{mm})$ including the disc diameter of $6 \mathrm{~mm}$; ${ }^{b}-$ mean of three analysis, \pm - standard deviation, ${ }^{*}$ - significant at $\mathrm{P}<0.05$.

Gram-positive bacteria have thicker peptidoglycan cell membranes compared to the Gramnegative bacteria, and it is harder for $\mathrm{CuO}$ to penetrate it, still our synthesized nanoparticles of both pure and capped nanoparticles shows the proper response for all the human pathogens. The extent of inhibition of bacterial growth reported in this study clearly shows the effect of capping on $\mathrm{CuO}$ nanoparticles.

\section{CONCLUSION}

In conclusion, the results evince that a simple noncomplex chemical precipitation method endued a facile and efficient route to synthesize PEG-capped $\mathrm{CuO}$ nanocrystal continuum of desired grades. However, the size of the particles were evidently influenced by the percentage of capping, principally proclaiming reduction in size of the nanoparticles as a result of capping $(13.06 \mathrm{~nm})$ when juxtaposed to their pristine counterpart. FESEM studies of PEG $(0.4 \mathrm{gm})$ capped particles show a considerable effect on the morphology and size distribution of the obtained artefacts. Comparing pristine $\mathrm{CuO}$, the antibacterial activity of $\mathrm{PEG}$ capped $\mathrm{CuO}$ nanorods developed a highest zone of inhibition for all the human pathogens. Obtained values of MIC for all the strains suggest that the prepared copper oxide nanoparticles shows excellent antibacterial activity and can be used as promising antibacterial agents in wide applications. Upon contemplating the above factors, $0.4 \mathrm{gm}$ of PEG as the capping agent is elicited as the optimum level for the preparation of $\mathrm{CuO}$ nanoparticles in order to control the size of the particle and for the morphology and also for the potent antibacterial applications.

\section{References:}

[1] R.S. Devan, R.A. Patil, J.H. Lin, Y.R. Ma., Appl. Func. Mater. 22 (2012) 3326-3370.

[2] H. Zhu, F. Zhao, L.Q. Pan., J. Appl. Phys. 101 (2007) 09H111-3, doi:10.1063/1.2711711.

[3] S. Sabbaghi1, Heydari Orojlou, M. R. Parvizi, R. Saboori, M. Sahooli., Int. J.Nano Dimens. 3(1) (2012) 69-73.

[4] K. T. Arulmozhi1, and N. Mythili., AIP ADVANCES 3, 122122 (2013).

[5] M.H. Huang, S. Mao, H. Feick., [J] Sience,292(2001)1897-1899.

[6] X.Y. Zhang, H.C. Ong., [J]. Chem 393(2004) 17- 21. 
[7] Vinay Kumar Patel and Shantanu Bhattacharya Appl. Mater. Interfaces 5(2013)13364-13374.

[8] Thi My Dung Dang, Thi Thu Tuyet Le, Eric Fribourg-Blanc, Mau Chien Dang., Adv. Nat. Sci: Nanosci. Nanotechnol. 2 (2011).

[9] C. C. Vidyasagar, Y. Arthoba Naik, T. G. Venkatesha, R. Viswanatha., Nano- Micro Lett. 4 (2) (2012)73-77.

[10] Qi Liu, Yongye Liang, Hongjiang Liu , Jianming Hongc, Zheng Xu., Materials Chemistry and Physics 98 (2006)519-522.

[11] Rashi Gusain and Om P. Khatri., Journal of Materials Chemistry A.1(2013)5612-5619.

[12] S.Z. Li, H. Zhang, Y.J. Ji, D.R. Yang., Nanotechnology.15(2004)1428-1432.

[13] A.W. Bauer, W.M.M. Kirby, J.C. Sherris and M. Turck., American J. Clin. Pathol.45, (1966) 493.

[14] S.M. Hammond and Lambert PA., Antimicrobial actions. London: Edward Arnld Ltd (1978) 8 -9.

[15] L.S. Birks, H. Friedman., J. Appl. Phys. 17(1946)687-692.

[16] Shanmugam Cholan, Nadana Shanmugam, Natesan Kannadasan, Kannadasan Sathishkumar, Kanthasamy Deivam., j mater res technol .3(3)(2014)222-227.

[17] S. Rahima, M. Sasani Ghamsaria, S. Radima., SciIran. 19(2012)948-53.

[18] G. Zou, H. Li, D. Zhang, K. Xiong, C. Dong, Y. Qian., J. Phys. Chem. B 110 (2006) 16321637.

[19] M. Heinlaan, A. Ivask, I. Blinova, H.C. Dubourguier, A. Kakru., Chemosphere 71(2008) $1308-1316$. 